\title{
Stimulus-independent thought depends on central executive resources
}

\author{
JOHN D. TEASDALE, BARBARA H. DRITSCHEL, MELANIE J. TAYLOR, LINDA PROCTOR, \\ CHARLOTTE A. LLOYD, IAN NIMMO-SMITH, and ALAN D. BADDELEY \\ MRC Applied Psychology Unit, Cambridge, England
}

\begin{abstract}
Stimulus-independent thoughts (SITs) are streams of thoughts and images unrelated to immediate sensory input. Four experiments examined the contribution of aspects of working memory to production of SITs. In Experiments 1 and 2, interventions that were targeted on, respectively, phonological and visuospatial components of working memory both interfered with production of SITs, but there was evidence that these tasks also made demands on central executive resources. Experiments 3 and 4 specifically examined the hypothesis that production of SITs and control of nonproceduralized tasks both depend on central executive resources, and so should show mutual interference. In Experiment 3, prior practice on pursuit rotor and memory tasks reduced the interference with SITs from concurrent task performance. In Experiment 4, randomness within a task involving random-number generation was less when SITs were being produced concurrently than it was when they were not. The results suggest that production of SITs depends on central executive resources.
\end{abstract}

In situations in which the demands to process information from external sources are low, we are likely to experience a flow of thoughts or images, the contents of which are quite unrelated to immediate sensory input. These mental events have been described as stimulus-independent thoughts (SITs) (Singer, 1966, 1988). Often experienced as daydreams (Singer, 1966), SITs may also involve content related to anxious worry (Borkovec, Robinson, Pruzinsky, \& DePree, 1983), depressive rumination (Beck, 1976), or the intrusive concerns that keep us awake at night (Borkovec \& Boudewyns, 1976).

Attempts to understand the cognitive processes involved in the production of SITs have reflected more general assumptions about the organization of information-processing resources. In pioneering studies (reviewed in Singer, 1988), Singer and Antrobus investigated SITs occurring during signal-detection tasks in the laboratory. Findings from these studies were interpreted within a model in which a common, limited-capacity, central cognitive operator dealt with information from both external (sensory-perceptual) and internal (memory) sources (Antrobus, 1968). Within this model, increasing the demand to process external, task-related information would be expected to lead to a reduction in the processing of the internal, memory-derived information involved in the production of SITs. Consistent with this analysis, Antrobus found a substantial negative linear relationship between the rate of information presentation in a tone-detection task and the frequency with which SITs were reported.

In the period since Antrobus and Singer's early studies, there has been clearer recognition of the deficiencies of

Correspondence should be addressed to J. D. Teasdale, MRC Applied Psychology Unit, 15 Chaucer Road, Cambridge CB2 2EF, England. information-processing models that account for limitations in performance in terms of restricted availability of generalpurpose processing capacity (e.g., Allport, 1980). In response, models have been proposed in which processing capacity, and memory, are distributed throughout the cognitive system in the form of semi-autonomous, special purpose, or content-specific modular subsystems (e.g., Baddeley, 1986; Barnard \& Teasdale, 1991; Fodor, 1983; Shallice, 1988). Of these approaches, the working-memory theory of Baddeley and colleagues (Baddeley, 1986; Baddeley \& Hitch, 1974) has been most frequently employed in attempts to understand the production of stimulusindependent thought and worry (e.g., Eysenck, 1982, p. 99; Levey, Aldaz, Watts, \& Coyle, 1991; Rapee, 1991, 1993; Teasdale, 1989; Teasdale, Proctor, Lloyd, \& Baddeley, 1993).

The Baddeley-Hitch approach to working memory proposes three main components: a central executive and two peripheral slave systems - the visuospatial sketchpad and the articulatory loop-phonological store system (hereafter called the phonological loop). The central executive's main functions involve the supervision, scheduling, and integration of information from different sources, and include the attentional control of action (Baddeley, 1986, chapter 10). The visuospatial sketchpad is a short-term memory store for maintaining and manipulating visuospatial images. The phonological loop is a short-term memory system specialized for storage of material in phonological (speech) code. All components of working memory are assumed to be of limited capacity.

Previous discussions of the role of working memory in the production of SITs have variously implicated the phonological loop and the central executive as important components (e.g., Levey et al., 1991; Rapee, 1991, 1993; Teasdale, 1989; Teasdale et al., 1993). However, no study has 
systematically examined the possible roles of different aspects of working memory in the production of naturally occurring SITs. Rapee (1993) reported a study examining the extent of interference with worrisome thoughts by tasks assumed to make different demands on the three components of working memory. He concluded that interference was greatest from tasks that made demands on aspects of the central executive and phonological loop, and suggested that these components of working memory were particularly important in the production of worry. However, the worrisome thoughts studied by Rapee were created intentionally by subjects to experimental instructions, rather than being more naturally occurring spontaneous mental events. It is likely that deliberate effort to produce worry to instruction will, itself, make substantial demands on the control and coordination resources of the central executive. Consequently, it may be difficult to generalize from Rapee's findings to more naturalistically occurring worry or stimulus-independent thought.

The strategy of the present study was to investigate the effects on naturally occurring SITs in normal subjects of interventions specifically related to each of the three components of working memory. Experiments used interference methodology; the extent to which tasks that were related to specific resources of working memory interfered with spontaneous SIT production was assumed to reflect the involvement of those resources in thought production. Experiment 1 focused on the phonological loop, Experiment 2 on the visuospatial scratchpad, and Experiments 3 and 4 on the central executive.

\section{EXPERIMENT 1}

If the phonological-loop system is a necessary component in the sequence of processes maintaining streams of stimulus-independent thought, occupying its limited capacity with tasks specifically targeted on that subsystem should substantially disrupt the flow of thoughts and reduce the frequency with which they will be reported when subjects are asked to report the contents of consciousness. Experiment 1 used two tasks previously shown to have relatively specific effects on the phonological loop (Baddeley, 1986, chapters 3 and 5). The first of these tasks involved remembering, by vocal rehearsal, short sequences of digits at rates that can be handled within the capacities of the phonological-loop subsystem. The second task comprised articulatory suppression, a procedure that occupies the articulatory loop without requiring subjects to retain information specifying a sequence of digits. Experiment 1 examined whether these two verbal tasks reduced reported experiences of SITs below the frequency reported when sitting quietly with no external task demands.

It was assumed that, in addition to their specific effects on the phonological-loop system, the verbal tasks might also make control and coordination demands on central executive resources. Any effect on SITs might therefore be the result of competition for the resources of the central executive, rather than for those of the peripheral memory store. In order to get some assessment of the extent to which the verbal tasks called on central executive resources, Experiment 1 also examined, separately, the effects of the verbal tasks on a concurrent task with no verbal component. Within the Baddeley-Hitch workingmemory theory, any interference with this task would be attributed to demands from the verbal tasks for central executive resources.

\section{Method}

\section{Subjects}

Twelve female subjects, age 32-45, from the Applied Psychology Unit subject panel participated.

\section{Design}

The effects of three different experimental treatments (quiet, digit load, and articulatory suppression) were compared in within-subject designs. Each subject received each of the experimental conditions in two phases, one phase measuring SITs, and the other measuring performance on the concurrent nonverbal task. Half of the subjects, randomly allocated, received the phases in the order SITs-nonverbal task, while the other half received the phases in the reverse order.

In the phase measuring SITs, each of the three experimental conditions was administered in blocks of an average duration of $120 \mathrm{sec}$ (range $=100-140 \mathrm{sec}$ ). Each subject received six blocks of each of the three conditions. Blocks were randomly allocated to experimental conditions, with the constraint that there should be equal numbers of blocks in each condition.

In the phase measuring performance on the nonverbal task, the subjects completed three parallel forms of the task under quiet, digit load, and articulatory suppression conditions. The order of the three parallel forms and the three experimental conditions was determined by a Latin square design.

\section{Measures}

Frequency of SITs. Each of the blocks described in the preceding section was terminated by the experimenter saying "stop." This occurred at an interval varying randomly between 100 and $140 \mathrm{sec}$ after the start of the block (mean interval $=120 \mathrm{sec}$ ). . On hearing "stop," the subjects were told to report "exactly what was passing through your mind just as you heard me say 'stop'." The subjects' reports were transcribed for subsequent categorization. It should be noted that the subjects were asked to report what they were thinking at the moment that they heard "stop," rather than to attempt to reconstruct, from memory, their thoughts throughout the block.

The mental contents reported on thought probes were categorized in terms of whether or not they indicated SITs, which were defined as thoughts with no apparent connection with the experiment or with the experimental situation (e.g., "wondering what we are going to have for lunch today" and "an image of a restaurant where we had a meal with the children two years ago")

Each thought report was categorized by the experimenter and an independent judge blind to the experimenter's categorization. Interjudge agreement was calculated by dividing the number of times both raters agreed in categorizing a report as a SIT by the sum of the times on which they agreed and the times that they disagreed (i.e., one rater categorizing as SIT, the other not). Agreement was $92 \%$.

For each of the three experimental conditions there were six blocks, each terminated by a thought probe. For each experimental condition, the total number of thought probes categorized as SITs, was calculated and used as a measure of the frequency of SITs. This measure could range from 0 to 6 .

The flexibility of closure test (Eskstrom, French, Harman, \& Dermen, 1976) was used as the nonverbal concurrent task. This requires the subject to identify a target geometrical form in a series of more complex forms, making a mark below each form to indicate whether or not the target was contained within it. The test yields a measure of the time taken to complete the task, and an error score. In its stan- 
dard form, the test contains 400 figures, 100 on each of four pages. We adapted this, using the first page for practice (half under digit load and half under articulatory suppression conditions), and the remaining three pages as separate parallel forms, one for each experimental condition.

\section{Procedure}

The experiment was conducted by a female experimenter in a sound-attenuated room. The subjects had their eyes closed during all procedures in which thoughts were sampled. Each subject's forward and backward digit span was first determined to check that their forward span exceeded the five-digit load used in the experiment. This was the case for all subjects. The subjects next practiced a 2-min block of each of the three experimental conditions, all with their eyes closed. In the quiet condition, the subjects were told just to "let the mind do what it wants to do." In the digit load condition, the experimenter read out a new set of five digits (1-5) every $10 \mathrm{sec}$, and the subjects repeated them continuously at the rate of three digits per sec. In the articulatory suppression condition, the subjects repeated continuously a constant set of digits, " $1,2,3,4,5$," at the rate of three digits per sec. To ensure comparability with the digit load condition, every $10 \mathrm{sec}$ the experimenter herself repeated this set at that rate. Each of the three practice intervals terminated with the experimenter saying "stop." The subject then practiced reporting whatever was passing through her mind at the moment that she heard the word "stop."

Next, the subjects practiced the flexibility of closure task under digit load and articulatory suppression conditions. The experiment proper then began, the subjects receiving either the flexibility of closure task or the thought-probe procedure, in random balanced order across subjects. After completing the first procedure, the subjects proceeded to the alternate procedure-thought probe or flexibility of closure, as appropriate.

\section{Results}

\section{Frequency of SITs}

Table 1 shows the mean frequency of SITs in the quiet, digit load, and articulatory suppression conditions. These conditions differed reliably in their effects on $\operatorname{SITs}[F(2,22)$ $\left.=19.57, M S_{\mathrm{e}}=1.41, p<.001\right]$. Comparison among treatment means showed that, compared with the quiet condition, SITs were reliably reduced in both articulatory suppression $[F(1,22)=20.02, p<.001]$ and digit load $[F(1,22)=36.28, p<.001]$ conditions, which themselves did not differ reliably $[F(1,22)=2.40, p>.1]$.

\section{Flexibility of Closure Test}

An analysis of variance (ANOVA) of the times to complete 100 items on this test showed a reliable effect of condition $\left[F(2,22)=12.94, M S_{\mathrm{e}}=325.87, p<.001\right]$. Compared with the quiet condition, performance was slowed

Table 1

Experiment 1: Effect of Experimental Conditions on Frequency of SITs and on Time to Complete 100 Items of the Flexibility of Closure Test

\begin{tabular}{|c|c|c|c|c|c|c|}
\hline & \multicolumn{6}{|c|}{ Condition } \\
\hline & \multicolumn{2}{|c|}{ Quiet } & \multicolumn{2}{|c|}{$\begin{array}{l}\text { Articulatory } \\
\text { Suppression }\end{array}$} & \multicolumn{2}{|c|}{ Digit Load } \\
\hline & $M$ & $\overline{S D}$ & $M$ & $\overline{S D}$ & $M$ & $S D$ \\
\hline$\overline{\text { SITs }}$ & 4.33 & 1.78 & 2.17 & 1.75 & 1.42 & 1.73 \\
\hline $\begin{array}{l}\text { Flexibility of } \\
\text { closure (sec) }\end{array}$ & 160 & 20 & 176 & 20 & 197 & 39 \\
\hline
\end{tabular}

Note- $M$, mean; $S D$, standard deviation. both by concurrent articulatory suppression $[F(1,22)=$ $5.06, p<.05]$ and by concurrent digit load $[F(1,22)=$ $25.78, p<.001$ ], performance being reliably slower in digit load than in articulatory suppression $[F(1,22)=$ $7.99, p<.01]$.

An ANOVA of the number of errors in this test yielded a nonreliable overall effect of experimental condition $[F(2,22)=3.11, p<.1]$. Mean errors in 100 items were as follows: quiet $=2.83$, articulatory suppression $=2.17$, and digit load $=1.42$. Overall, error rates were very low, and there was a trend for the slowing in the two experimental conditions to be accompanied by greater accuracy.

\section{Discussion}

Two experimental conditions specifically targeted on the phonological-loop subsystem - articulatory suppression and retaining a 5-digit load by overt verbal rehearsalboth reduced the reported frequency of SITs compared with a control condition in which no task was performed. These results are consistent with the suggestion that the phonological loop is involved in the production of such SITs. However, both these verbal tasks also reliably interfered with performance on a concurrent nonverbal task. Such interference suggests that the digit load and articulatory suppression tasks made demands on more general processing resources, in addition to their demands on specifically phonological resources. It follows that the present pattern of results, although consistent with involvement of the phonological loop in SIT production, is also open to a number of alternative explanations. For example, production of such thoughts might require general-purpose cognitive resources, and the observed interference with thought production by the verbal tasks might reflect the demands of these tasks for these more general, not specifically phonological, resources.

As a way of investigating this issue further, Experiment 2 examined the effects on SITs of a simple spatialmotor tapping task which appeared to make no demands on the phonological-loop subsystem (Farmer, Berman, \& Fletcher, 1986), but which had similar rate characteristics to the articulatory suppression and digit load conditions. If this spatial-motor task has effects on SITs similar to those observed in Experiment 1, it would suggest that interference with the phonological-loop subsystem of working memory is not a necessary requirement for interrupting the flow of SITs.

\section{EXPERIMENT 2}

Experiment 2 examined the effects of three experimental conditions (quiet and two versions of a simple spatialmotor task) on the frequency of SIT reports, and on a concurrent semantic decision task, the silly sentences test (Baddeley, Eldridge, Lewis, \& Thomson, 1984). The design used was the same as that used in Experiment 1. The silly sentences test, rather than the flexibility of closure test, was used as the concurrent task because it makes minimal demands on spatial-perceptual processing. Consequently, any disruption of concurrent task performance by the spa- 
tial-motor task is likely to be attributable to demands on more central resources, rather than simply to competition for the peripheral visuospatial sketchpad.

Method
Subjects
Twelve female subjects, age 37-50, from the Applied Psychology
Unit subject panel participated.

\section{Measures}

Frequency of SITs. This was measured as in Experiment 1. Interjudge agreement on the categorization of thoughts as SITs was $84 \%$.

Silly sentences test. Each of the five parallel forms of this test consists of 50 sentences (e.g., "Bishops can be bought in shops"), half of which are true and half of which are not. The subject is asked to judge as quickly as possible whether each sentence is true or not. Time to complete the 50 sentences was recorded with a stopwatch, and the number of errors was noted.

\section{Procedure}

The procedure was essentially similar to that of Experiment 1 . In the practice phase, the subjects completed one of the parallel forms of the silly sentences task in the quiet condition, and half of a second parallel form in each of the two tapping conditions.

The spatial-motor task required subjects to tap on four raised 17 . $\mathrm{mm}^{2}$ keyboard switches arranged in a square $(35 \times 35 \mathrm{~mm})$, using the first or second finger of the writing hand. In the taps-same condition, the subjects tapped the keys in a continuous clockwise sequence, the experimenter saying "same" every $10 \mathrm{sec}$. In the taps-change condition, the subject began tapping in a clockwise direction. Every $10 \mathrm{sec}$, the experimenter said either "same" or "change" in random sequence. If she said "same," the subject continued tapping as before; if she said "change," the subject changed direction (i.e., from clockwise to counterclockwise, or vice versa). Tapping rate was three taps per sec, the same rate of performance as that used in the articulatory suppression and digit load conditions in the previous experiment. The task was modeled and practiced until tapping rates were accurate. Subsequently, rates were periodically checked to ensure that the subjects were conforming to the assigned rates.

In the thought-sampling section of the experiment, the subjects performed the tapping task with their eyes closed. In the silly sentences section of the experiment, the subjects had their eyes open, but their visual attention was focused on the written silly sentences. Because the subjects' writing hands were used in the tapping task, the form of the silly sentences task used in this experiment required the subjects to say, rather than write, whether each sentence was true or not; the experimenter recorded their answers.

\section{Results}

\section{Frequency of SITs}

Table 2 shows the mean frequency of SIT reports in the quiet, taps-same, and taps-change conditions. The experimental conditions differed reliably in their effects on SITs $\left[F(2,22)=18.01, M S_{\mathrm{e}}=1.48, p<.001\right]$. Comparison among treatment means showed that, compared with the quiet condition, both taps-same $[F(1,22)=27.02, p<$ $.001]$ and taps-change $[F(1,22)=27.02, p<.001]$ reliably reduced the frequency of SITs, but did not themselves differ, scores being identical in the two conditions.

\section{Silly Sentences}

Times taken to complete 50 silly sentences in the quiet, taps-same, and taps-change conditions are shown in Table 2 , and differed reliably $\left[F(2,22)=12.48, M S_{\mathrm{e}}=\right.$
Table 2

Experiment 2: Effect of Experimental Conditions on Frequency of SITs and on Time to Complete 50 Silly Sentences

\begin{tabular}{|c|c|c|c|c|c|c|}
\hline & \multicolumn{6}{|c|}{ Condition } \\
\hline & \multicolumn{2}{|c|}{ Quiet } & \multicolumn{2}{|c|}{ Taps-Same } & \multicolumn{2}{|c|}{ Taps-Change } \\
\hline & $M$ & $S D$ & $M$ & $S D$ & $M$ & $S D$ \\
\hline SITs & 4.58 & 1.38 & 2.00 & 1.86 & 2.00 & 1.91 \\
\hline Silly & & & & & & \\
\hline sentences $(\mathrm{sec})$ & 69 & 10 & 79 & 14 & 86 & 19 \\
\hline
\end{tabular}

Note- $M$, mean; $S D$, standard deviation.

$70.91, p<.001]$. Compared with the quiet condition, both taps-same $[F(1,22)=8.60, p<.01]$ and taps-change $[F(1,22)=24.70, p<.001]$ reliably slowed performance, taps-change itself producing marginally slower performance than taps-same $[F(1,22)=4.15, p<.1]$. Error rates were low in all conditions $[M=1.00$ (quiet), 0.75 (taps-same), and 1.50 (taps-change), and did not differ between conditions.

\section{Discussion}

The spatial-motor tapping task, targeted on the visuospatial sketchpad, produced a substantial and reliable reduction in the frequency of SITs. However, the tapping task also produced reliable interference in a task (silly sentences) which appeared to make few demands on the sketchpad system. Analogously, in Experiment 1, digit load and articulatory suppression, targeted on the phonologicalloop subsystem, produced reliable interference both in SITs and in a task (flexibility of closure) which appeared to make no demands on the phonological-loop system. Such findings suggest that the verbal and tapping tasks studied in Experiments 1 and 2 placed demands on more central aspects of working memory, in addition to demands on their respective specific subsystems. It is possible, therefore, that the interference in thought production observed in Experiments 1 and 2 reflects the involvement in SIT production of more general, central resources of working memory, in addition to any more specific peripheral components.

Within the Baddeley-Hitch theory, general-purpose cognitive resources are included within the central executive, the least well-specified of the components of working memory. To date, the central executive's most clearly specified function has been the control and coordination of performance. It is assumed that the central executive has limited resources that coordinate task performance through the selection, facilitation, and control of subprocesses which are themselves shared by a number of tasks. If these limited resources are also required for the production of SIT, mutual interference between task coordination and SIT production would be expected.

In general, the demands on the control and coordinating functions of the central executive from task performance will be greater when a task is novel than when, following practice, some degree of automatization has occurred. Experiment 3 examined the effects of prior practice of a task on the extent to which the task interfered with SIT production. Extended practice of a novel task would be ex- 
pected to reduce the demands on central executive resources for the control and coordination of task performance. Consequently, it was predicted that if SIT production depends on these same limited resources, prior practice on tasks would reduce the extent to which they interfered with SIT production; frequency of SITs should be greater during equivalent performances of previously practiced tasks than during previously unpracticed tasks.

\section{EXPERIMENT 3}

\section{Method}

\section{Subjects}

Thirty-two female subjects (mean age $=46.8$ years, $S D=7.5$ ) from the Applied Psychology Unit subject panel participated.

\section{Design}

The subjects were randomly allocated to two equal groups. In the practice phase, one group practiced a pursuit rotor task but not a memory task, and the other group practiced a memory task but not a pursuit rotor task. In the performance phase, all subjects performed on both the pursuit rotor and memory tasks. Within each of the two groups, half of the subjects, randomly allocated, performed the pursuit rotor task first and the memory task second in the performance phase, and the remaining half of the subjects performed the memory task first and the pursuit rotor task second in that phase.

\section{Procedure}

The experiment was conducted in a sound-attenuated darkened room by a female experimenter.

Practice at thought report. The subjects received three practice trials at thought report. They sat quietly with their eyes closed for three periods averaging 1 min each. At the end of each period, they were asked to report what was going through their mind at the precise moment that the experimenter terminated the trial by saying "stop."

Task-practice phase. The subjects then practiced one of two tasks for 12 trials, the trial length averaging $90 \mathrm{sec}$ (range $=70-110 \mathrm{sec}$ ). A tape recording was used to begin and end each trial. At the end of each trial, the subjects reported what was passing through their mind at the moment that they heard the word "stop" that terminated the trial. The experimenter recorded the subjects' thought reports verbatim.

Pursuit rotor task. The subjects tracked a light moving in a circle (15-cm radius) at 20 revolutions per min on a Forth Instruments Pursuit Rotor, using a light-sensitive stylus. The subjects received no feedback on performance, other than that available by visual inspection, but accuracy of performance (as a percentage of total time on target) was recorded by the experimenter at the end of each trial. The subjects chose whether to sit or stand during the task, knowing that they would be performing for approximately $30 \mathrm{~min}$ in the first phase of the experiment.

Memory task. The subjects were required to remember single digits (0-9) presented in randomly determined order at intervals of $4 \mathrm{sec}$ from a tape recorder. Each digit was to be remembered until a new digit was heard, at which point the old digit was to be disregarded and the new digit retained. As a check on compliance with instructions, at irregular intervals the subjects were asked to state the digit they were remembering. Intervals were randomly determined in terms of the number of digits presented within them; they averaged 4.5 digits.

Performance phase. In this phase, all of the subjects completed six trials (average duration $=90 \mathrm{sec}$ ) on each of the two tasks-pursuit rotor and memory -in counterbalanced order. For half of the subjects, pursuit rotor had previously been practiced in the practice phase but memory had not. For the other half of the subjects, memory had previously been practiced in the practice phase but pursuit rotor had not. The beginning and end of each trial were indicated by a tape recorder, which also presented the digits in the memory task.
The subjects made thought reports at the end of each of the 12 trials in the performance phase. At the end of the experiment, the subjects were asked about what they perceived the purpose of the experiment to have been, after which they were thanked and paid.

Categorization of thought content. Transcriptions were made of the mental contents that the subjects reported experiencing at the point at which they heard the word "stop" that terminated each trial. As in Experiments 1 and 2, these reports were categorized by the experimenter and an independent judge blind to the subject's experimental condition in terms of whether or not they indicated the presence of SITs. Scores reflecting frequencies of SITs were derived by counting the number of trials on which SITs were reported on Trials $1-3,4-6,7-9$, and $10-12$ of the performance phase for each of the 32 subjects. Interrater agreement on these 128 scores was perfect in 103 cases $(81 \%)$, differed by one score point in 23 cases $(18 \%)$, and differed by two score points in the remaining two cases $(2 \%)$. The scores from the blind independent rater were used in the analyses reported.

\section{Results}

\section{Accuracy of Task Performance}

The logic of the present experiment requires that subjects who have previously practiced a task should perform the task during the performance phase at a level that is at least as good as subjects who have not previously practiced the task. Otherwise, any excess of SITs shown by practiced subjects when performing the task could be attributed to poorer quality of task performance, rather than to any reduction in the allocation of central executive resources as a result of prior practice.

Memory task. Recall during the performance phase was perfect for 28 out of the 32 subjects; the 4 subjects showing errors were divided equally between the group that had previously practiced this task and the group that had not.

Pursuit rotor. An ANOVA of the percentage of time on target during the performance phase, with previous practice versus no previous practice as a between-subject factor and phase (Trials $1-3$ vs. Trials $4-6$ ) as a withinsubject factor, revealed reliably superior performance for the practiced group $(M=68.13, S D=18.17)$ compared with the nonpracticed group $[M=50.28, S D=10.08$; $\left.F(1,30)=13.45, M S_{\mathrm{e}}=378.72, p<.001\right]$, and a reliable interaction between phase and practice $[F(1,30)=6.67$, $\left.M S_{\mathrm{e}}=337.64, p<.02\right]$. For practiced subjects, performance deteriorated from Trials $1-3(M=72.00, S D=$ $14.47)$ to Trials $4-6(M=64.25, S D=20.99)$, as is typical for practiced subjects tested under conditions of massed practice (Bilodeau \& Bilodeau, 1961), whereas performance improved slightly from Trials $1-3(M=49.56, S D=$ $7.51)$ to Trials $4-6(M=51.00, S D=12.34)$ for nonpracticed subjects.

The overall superior performance of the group with prior practice on the pursuit rotor, together with the equivalent, virtually perfect performance of the two groups on the memory task, means that any excess of SITs during the performance phase associated with previous practice cannot simply be attributed to poorer performance on the primary task.

\section{Frequency of SITs in Performance Phase}

The frequencies with which SITs were reported at thought probes during the performance phase are shown 
in Figure 1. A $2 \times 2 \times 2$ repeated measures ANOVA on these data examined the between-subject effect of group (practiced pursuit rotor task vs. practiced memory task) and the within-subject effects of practice (the task that the subject practiced previously vs. the task that the subject had not previously practiced) and phase within a task (the first three trials vs. the second three trials).

The only reliable effects revealed by the ANOVA were a main effect of practice $\left[F(1,30)=14.23, M S_{\mathrm{e}}=.752\right.$, $p<.001]$ - there being more SITs during the task a subject had previously practiced than during the task the subject had not practiced - and a main effect of phase $[F(1,30)$ $\left.=11.40, p<.01, M S_{\mathrm{e}}=.247\right]$-there being more SITs during the second three trials of a task than during the first three trials. Separate analyses for the group that had practiced the pursuit rotor task and the group that had practiced the memory task both showed reliable main effects of practice within each group $\left[F(1,14)=9.76, M S_{\mathrm{e}}=\right.$ $.578, p<.01$; and $F(1,14)=7.56, M S_{\mathrm{e}}=.670, p<.02$, respectively].

\section{Postexperimental Inquiry}

No subject gave any indication that she had thought that the purpose of the experiment was to study the effects of practice on the extent to which a task interfered with SITs.

\section{Discussion}

The results confirmed the prediction that previous practice on a task will reduce the extent to which performance on the task interferes with production of reportable SITs. Effects of practice reducing the interference with SIT production from a concurrent task were observed in both a verbal memory task and a perceptual-motor skill task. This pattern of results is more parsimoniously explained by the suggestion that the effects of practice are mediated through reductions in demand for central resources of control and coordination, common to the two tasks, rather than through reductions in demand

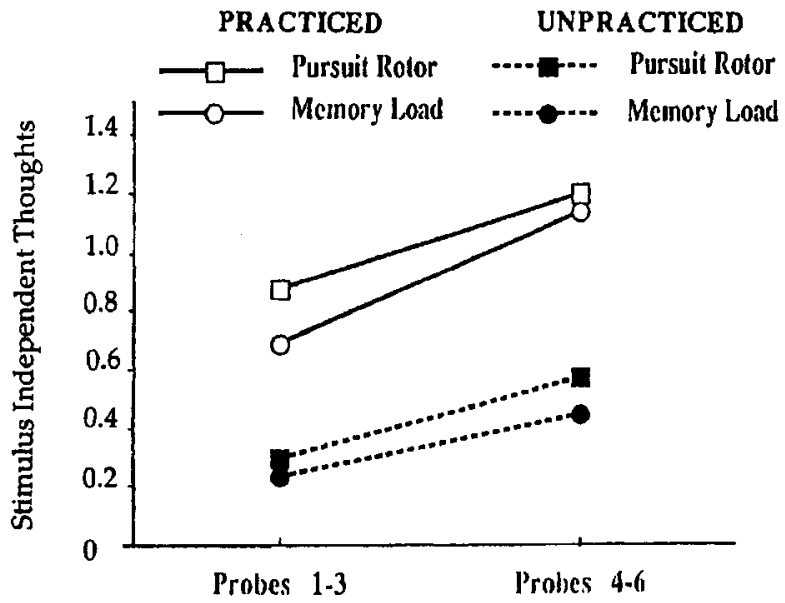

Figure 1. Experiment 3: Frequency with which SITs were reported during performance phase. for more specialized verbal or spatial processing resources, on which the two tasks would differ.

Experiment 4 involved a further specific test of the hypothesis that production of SITs depends on central executive resources.

\section{EXPERIMENT 4}

Baddeley (1986, chapter 10) suggested that the task of generating random sequences of letters (or numbers) can be considered a paradigmatic example of a task requiring high levels of central executive resources of control and coordination. In this task, in order to produce random patterns, control resources have to monitor and override habitual tendencies to produce sequences of letters or numbers that are strongly associated (e.g., ascending or descending series, odd or even numbers). The randomness of the sequences of letters or numbers produced can be used as an indicator of the extent to which limited central resources of control and coordination have been utilized in the randomization task; the more of these resources that are deployed on other concurrent tasks, the less random will be the sequences produced.

Experiment 4 examined the randomness of numbers generated in periods immediately preceding occasions on which SITs were reported compared with periods immediately preceding occasions on which no thoughts were reported. It was predicted that if SIT production and random-number generation compete for the same central executive resources, then, given a primary task of generating random sequences of numbers, thought probes on which concurrent SITs are reported should be preceded by lower levels of randomness than thought probes indicating an absence of task-irrelevant thoughts.

\section{Method}

\section{Subjects}

Twenty-six female subjects (mean age $=43.6$ years, $S D=6.1$ ) from the Applied Psychology Unit subject panel participated.

\section{Measures}

Mental contents. As in the previous experiments, the mental contents reported by the subjects at thought probes were written down verbatim and subsequently categorized by the experimenter and an independent judge. SITs were defined in the same way as in the previous experiments. Interjudge agreement on categorization of SITs was $93 \%$. Mental contents were categorized as no thoughts (NTs) when subjects reported either that their minds were blank, that they were merely aware of sensations or perceptions related to current sensory input, or that they were merely aware, as sound or image, of the number currently being produced. If there was any internal commentary on sensations or on task performance, or any further elaboration of images, such contents were not categorized as NTs. Interjudge agreement in categorizing NTs, calculated by dividing the number of times the two judges agreed in categorizing a report as an NT by the sum of the times on which they agreed and on which they disagreed, was $90 \%$.

Randomness of numbers generated. As an index of the randomness of the 100 digits generated prior to each thought probe, Evans's (1978) index of randomisation (RNG) was calculated. RNG was adapted from Tulving's (1962) subjective organisation index of clustering in free recall, and takes account of overusage of repeated pairs 
or sequences of digits. Responses are entered into a $10 \times 10$ matrix reflecting the frequency with which any digit follows any other digit in 100 consecutive responses. With $f_{i}$ as the marginal frequency for row $(i)$, and $f_{i j}$ as the frequency in Cell ${ }_{i j}$ (where $j$ indicates column),

$$
\mathrm{RNG}=\sum f_{i j} \log f_{i j} / \sum f_{i} \log f_{i} \text { (Evans, 1978, p. 36). }
$$

Here, $f_{i}$ indicates the frequency of the response $i$ among the responses in a random-digit-generation task; $f_{i j}$ indicates the number of times the bigram $i j$ occurs (i.e., the number of times that $i$ is followed by $j$ ). The numerator represents the amount of predictability in the way in which bigrams occur as pairs of consecutive responses. The denominator represents the amount of predictability in the way in which the 10 digits occur as responses. The ratio RNG is thus a measure of the amount of predictable or stereotypical pattern (or lack of randomness) in the bigrams relative to that in the frequencies with which the 10 digits actually occur in the responses. Higher values of RNG correspond to lower randomness. RNG can range in value from 0 to 1 . Evans (1978) reported mean RNG, from a number of samples of subjects, based on 100 numbers produced at one per sec, as $.300(S D=.045)$.

In the present experiment, RNGs based on 100 numbers produced at one per sec can only provide an average index of randomness for an interval nearly $2 \mathrm{~min}$ long. In order to get a measure more closely related in time to thought probes, a measure of high-frequency bigrams was calculated as follows: The matrices for the 20 sets of 100 digits from each subject were collapsed into an overall $10 \times 10$ matrix, from which the sequences of two digits occurring with high frequency ( 30 or more times) in the experiment overall, for that subject, could be identified. The number of such high-frequency bigrams occurring in the 20 numbers preceding a thought probe was then counted, to give a measure of randomness (or lack of randomness) based on a sample of 20 -sec duration. If in any block of 20 numbers the subject generated a number other than 1-10 (e.g., 12), an arbitrary score equal to the maximum number of common bigrams in any of the blocks being compared for that subject was entered. This procedure was adopted on the assumption that production of a nontarget number represented an even greater lack of attentional control than generation of nonrandom target numbers.

\section{Procedure}

The experiment was conducted in a sound-attenuated room by a female experimenter. The subjects sat with their eyes closed for 20 intervals $($ mean duration $=110 \mathrm{sec}$, range $=100-120 \mathrm{sec}$ ). They were told:

During each interval you will be asked to say one digit between 1 and 10 inclusive, out loud, every second. The order in which you say the digits should be random. What I mean by that is that each digit should have an equal chance of being selected each time. It's rather like throwing a 10 sided die each time, one with the numbers 1 to 10 on the sides. Although it might not happen on a few throws, over a large number of throws each number would occur as often as every other number and in no particular order. Remember to use all numbers from 1 to 10 inclusive.

The pace of one digit per sec was set by a metronome. The experimenter wrote down the numbers as subjects said them, and terminated each interval by saying "stop." At this point, the subjects reported mental contents, and rated how aware they were of the numbers that they were saying, using a scale marked not at all aware, slightly aware, moderately aware, very aware, extremely aware.

\section{Results}

All results are based on within-subject comparisons using dependent $t$ tests.

\section{Randomness Measures in Blocks Preceding SIT and NT Reports}

In view of the demanding nature of the task involving random-number generation, it had been anticipated that overall frequency of SIT reports would be low. For this reason, an a priori criterion for inclusion in the analysis of randomness had been set. This was that subjects had to have at least four blocks of number-generation preceding probes at which SITs were reported and four blocks of numbergeneration preceding probes on which NTs were reported. In this way, subjects were included in analyses of randomness only when the means for measures of randomness preceding each type of thought report were based on at least four samples. Fourteen subjects met this criterion.

Index of randomization (RNG). For each subject in the subsample, mean RNG for the blocks of 100 numbers preceding SIT and NT reports were calculated. Consistent with prediction, mean RNG was reliably greater in blocks preceding SIT reports $(M=.329, S D=.036)$ than it was in blocks preseding NT reports $[M=.317, S D=.029$; dependent $t(13)=2.77, p<.01$, one-tail], indicating greater randomness in the latter case. As an indication of the representativeness across subjects of these differences in group means, the direction of the differences in means within individual subjects showing differences of .010 or greater was examined. Among these 9 subjects, mean RNG was greater preceding SIT reports than it was preceding NT reports in 8 subjects, only 1 subject showing the reverse pattern (sign test: $p=.02$, one-tail).

High-frequency bigrams. Also consistent with the prediction, the mean number of high-frequency bigrams was reliably greater in the 20 digits preceding SIT reports $(M=$ $8.81, S D=1.50$ ) than it was in the 20 digits preceding NT reports $[M=7.99, S D=1.70$; dependent $t(13)=2.62$, $p<.02$, one-tail], indicating greater randomness in the latter case. As an indication of the representativeness across subjects of these differences in group means, the direction of the differences in means within individual subjects showing differences of more than .50 was examined. Among these 11 subjects, bigram frequency was greater preceding SIT reports than it was preceding NT reports in 10 subjects, only 1 subject showing the reverse pattern (sign test: $p=.006$, one-tail).

\section{Awareness of Digits}

For each of the total sample of 26 subjects, the 20 blocks were divided as close as possible to a median split on subjects' ratings of their awareness of the digits that they were generating. The proportions of high- and low-awareness blocks followed by SITs were calculated. Compared with low-awareness blocks, high-awareness blocks were associated with reliably fewer reports of SITs [high awareness: $M=12 \%, S D=20$; low awareness: $M=37 \%, S D=$ 28 ; dependent $t(25)=5.07, p<.001]$.

\section{Discussion}

Randomness of numbers produced in a task involving random-number generation was lower in intervals immediately preceding thought probes on which SITs were reported than it was in intervals preceding probes on which no task-irrelevant thoughts were reported. This result was predicted by the hypothesis that random-number generation and production of SITs compete for the same, limited 
control and coordination resources of the central executive; when more control resources are allocated to production of SITs, fewer resources are available to control the generation of random numbers and, consequently, randomness decreases.

The finding that SITs were less frequent when subjects reported being highly aware of the digits they were producing replicates and extends earlier findings. Teasdale et al. (1993, pp. 429-430) found that subjects reported reliably fewer SITs at times when they rated themselves highly aware of numbers they were remembering than they did when they reported themselves as less aware, even though task performance was very similar under the two conditions. The negative relationship between awareness of task stimuli and reports of SITs can be explained by the suggestion (e.g., Norman \& Shallice, 1986) that attentional control of action is associated with conscious awareness. On this view, reports of high subjective awareness of task stimuli can be used as a marker that central control resources have been deployed to task management. The association between high awareness of task stimuli and reduced production of SITs can be explained in terms of the competition for control resources between SIT production and attentional control of task performance; compared with conditions of low task awareness, under conditions of high task awareness, fewer central resources are available for SIT production as a result of the deployment of more of those resources to task management.

Experiment 4 provided evidence of a reliable, predicted relationship between aspects of self-reported mental events and an index derived from behavioral performance on a number-generation task. This finding provides objective evidence for the validity of the subjective self-report measures used in this and the preceding experiments.

\section{GENERAL DISCUSSION}

The evidence from Experiments 3 and 4 provides specific support for the hypothesis that production of SITs depends on central executive resources. Experiment 3 used an experimental manipulation (prior practice) that would be expected to reduce the demand for central executive resources for task control and coordination. This manipulation produced reductions in the interference with SIT production by both a verbal memory task and a spatial, perceptual-motor skill task. In Experiment 4, within-task variations in the deployment of central executive resources to the task of generating random numbers (indicated by variations in the randomness of the numbers produced) were associated with variations in reports of SITs; reports of SITs predicted lower levels of randomness on the number-generation task. The pattern of results from both experiments is consistent with the suggestion that SIT production and the control and coordination of nonproceduralized tasks compete for the same, limited central executive resources, and that such resources are necessary for the production of SITs.

The central executive is the least well-specified component of the Baddeley-Hitch approach to working mem- ory. Consequently, the present results are probably best interpreted as supporting the hypothesis that SIT production depends on central executive resources of control and coordination, as these resources would be characterized both within that model and more generally.

The conclusion that production of SITs depends on central executive resources constrains the type of accounts that can be offered to explain the production of streams of stimulus-independent thoughts and images. Teasdale and Barnard (1993, pp. 160-168) offer an analysis that necessarily implies the type of mutual interference observed in the present experiments between SIT production and task performances that make demands on central executive resources. This analysis was developed within a wider, comprehensive information-processing approach (interacting cognitive subsystems [ICS]; Barnard \& Teasdale, 1991). The ICS account suggests that production of connected sequences of SITs depends on continuing reciprocal exchanges and transformations of information between two specialized cognitive subsystems handling, respectively, information at the level of specific, propositional meanings, and a more generic level of representation related to schematic mental models. Within the ICS approach, central executive functions of control and coordination are also realized by continuing cycles of reciprocal interaction, exchange, and transformation between the two subsystems dealing with representations at the level of specific meanings and the more generic level of schematic mental models (Teasdale \& Barnard, 1993, pp. 78-81). From this analysis, mutual interference between central executive control of tasks and production of SITs, as observed in the present experiments, would be expected. This is because, within the ICS approach, the transformation processes mediating the interactions between representations of these different types of information can handle only one coherent data stream at a time.

The present investigations also have implications for the design of interventions directed at the short-term control of unwanted SITs, such as those important in worry and insomnia. The findings suggest that the most effective distraction tasks to block unwanted thoughts will be those that make continuous demands on the control and coordinating resources of the central executive, but that do not, themselves, generate SITs. This analysis suggests that a relatively simple articulatory suppression task, such as continuously repeating "the, the, the," might have only limited effectiveness, which would decline with practice as the task became automated. To make this a more effective distraction task, it would be necessary to incorporate a greater demand on control and coordination resources. Interestingly, this is exactly what Levey et al. (1991) found in a clinical study of methods for dealing with unwanted thoughts preventing sleep. Simple repetition of "the, the, the" had only limited effects. However, when subjects were așked to generate "the" at constantly varying random intervals, the effectiveness of the procedure improved considerably. Clearly, the latter variation of the task would make many more, and continuing, demands on central executive resources, much as in the task of generating random numbers. 


\section{REFERENCES}

ALLPORT, D. A. (1980). Attention and performance. In G. Claxton (Ed.), Cognitive psychology: New directions (pp. 112-153). London: Routledge \& Kegan Paul.

ANTROBUS, J. S. (1968). Information theory and stimulus-independent thought. British Journal of Psychology, 59, 423-430.

BADDELEY, A. D. (1986). Working memory. Oxford: Oxford University Press.

Baddeley, A. D., Eldridge, M., LewIS, V., \& Thomson, N. (1984). Attention and retrieval from long-term memory. Journal of Experimental Psychology: General, 113, 518-540.

Baddeley, A. D., \& Hitch, G. J. (1974). Working memory. In G. H. Bower (Ed.), The psychology of learning and motivation (Vol. 8, pp. 47-89). New York: Academic Press.

BaRnaRD, P. J., \& TeasDale, J. D. (1991). Interacting cognitive subsystems: A systemic approach to cognitive-affective interaction and change. Cognition \& Emotion, 5, 1-39.

BECK, A. T. (1976). Cognitive therapy and the emotional disorders. New York: International Universities Press.

Bilodeau, E. A., \& Bilodeau, I. M. (1961). Motor skills learning. Annual Review of Psychology, 12, 243-280.

Borkovec, T. D., \& BoudewYNS, P. A. (1976). Treatment of insomnia with stimulus control arrd progressive relaxation procedures. In J. D. Krumbolz \& C. E. Thoresen (Eds.), Counselling methods (pp. 328344). New York: Holt, Rinehart \& Winston.

Borkovec, T. D., Robinson, E., Pruzinsky, T., \& DePree, J. A. (1983). Preliminary exploration of worry: Some characteristics and processes. Behaviour Research \& Therapy, 21, 9-16.

Eskstrom, R. B., French, J. W., Harman, H. H., \& Derman, D. (1976). Manual for kit of factor-referenced cognitive tests. Princeton, NJ: Educational Testing Service.

Evans, F. J. (1978). Monotoring attention deployment by random number generation: An index to measure subjective randomness. Bulletin of the Psychonomic Society, 12, 35-38.

EYSENCK, M. W. (1982). Attention and arousal: Cognition and performance. Berlin: Springer-Verlag.
Farmer, E. W., Berman, J. V. F., \& Fletcher, Y. L. (1986). Evidence for a visuo-spatial scratch-pad in working-memory. Quarterly Journal of Experimental Psychology, 38A, 675-688.

FODOR, J. (1983). The modularity of mind. An essay on faculty psychology. Cambridge, MA: MIT Press, Bradford Books.

Levey, A. B., Aldaz, J. A., Watrs, F. N., \& Coyle, K. (1991). Articulatory suppression and the treatment of insomnia. Behaviour Research \& Therapy, 29, 85-89.

Norman, D. A., \& Shallice, T. (1986). Attention to action: Willed and automatic control of behaviour. In G. E. Schwartz \& D. Shapiro (Eds.), Consciousness and self-regulation: Advances in research and theory (Vol. 4, pp. 1-18). New York: Plenum.

RAPEE, R. M. (1991). Generalized anxiety disorder: A review of clinical features and theoretical concepts. Clinical Psychology Review, 11 419-440.

RAPEE, R. M. (1993). The utilisation of working memory by worry. Behaviour Research \& Therapy, 31, 617-620.

SHALLICE, T. (1988). From neuropsychology to mental structure. Cambridge: Cambridge University Press.

SINGER, J. L. (1966). Daydreaming. New York: Random House.

SiNGER, J. L. (1988). Sampling on-going conscioüsness and emotional experiences: Implications for health. In M. J. Horowitz (Ed.), Psychodynamics and cognition (pp. 297-346). Chicago: University of Chicago Press.

Teasdale, J. D. (1989). Daydreaming, depression, and distraction. The Psychologist, 2, 189-190.

TEASDALE, J. D., \& BARNARD, P. J. (1993). Affect, cognition, and change: Remodelling depressive thought. Hove, U.K.: Erlbaum.

Teasdale, J. D., Proctor, L., Lloyd, C. A., \& Baddeley, A. D. (1993). Working memory and stimulus-independent thought: Effects of memory load and presentation rate. European Journal of Cognitive Psychology, 5, 417-433.

TuLviNG, E. (1962), Subjective organization in free recall of "unrelated" words. Psychological Review, 69, 344-354.

(Manuscript received April 20, 1994; revision accepted for publication October 17, 1994.) 